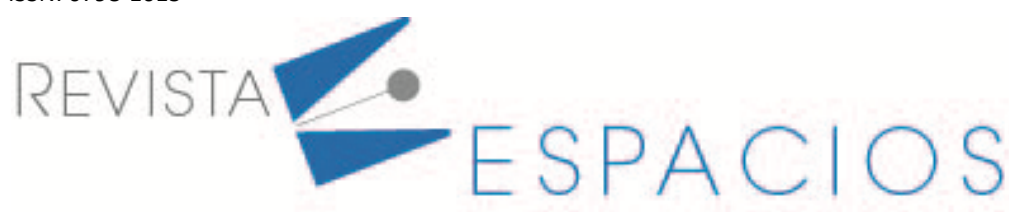

Vol. 42 (06) $2021 \cdot$ Art. 4

Recibido/Received: 04/12/2020 • Aprobado/Approved: 03/03/2021 • Publicado/Published: 30/03/2021

\title{
Logística urbana de la ciudad de Tulcán, Ecuador: diagnóstico y análisis
}

\section{Urban logistics of the Tulcán city, Ecuador: diagnosis and analysis}

\author{
BELTRÁN, Daniel M. ${ }^{1}$ \\ MAFLA, Iván $\mathrm{G}^{2}$ \\ MORA, Edwin J. ${ }^{3}$
}

\begin{abstract}
Resumen
Teniendo en cuenta los actores principales de la dinámica de la logística urbana (sistema público, proveedores y consumidores) en el presente trabajo se realiza una caracterización que parte de la zonificación de la ciudad de Tulcán, Ecuador, basada en las características de uso del suelo por sector, para posteriormente, mediante instrumentos de recolección de información, como registros, encuestas y entrevistas, determinar los generadores y atractores de viajes de cada una de las zonas definidas, identificando sus frecuencias, con lo cual se determina que los dinamizadores de viajes son los proveedores de productos de consumo masivo y sus clientes.
\end{abstract}

Palabras clave: logística urbana, optimización, diagnóstico.

\begin{abstract}
Taking into account the main actors of the dynamics of urban logistics (public system, suppliers and consumers), in this work a characterization is carried out that starts from the zoning of the city of Tulcán, Ecuador, based on the characteristics of land use. by sector, for later, by means of information gathering instruments, such as records, surveys and interviews, to determine the trip generators and attractors of each of the defined areas, identifying their frequencies, with which it is determined that the trip drivers are suppliers of consumer products and their customers.
\end{abstract}

Key words: urban logistics, optimization, diagnosis.

\section{Introducción}

El crecimiento poblacional ha ocasionado la escasez de recursos o la dificultad de conseguirlos, esto unido a que la desvalorización del trabajo agrícola ha provocado que las personas se vean obligadas a migrar a las ciudades para conseguir trabajo y poder cubrir las necesidades básicas. Esta migración conlleva al incremento demográfico mismo que a su vez genera varios problemas como la utilización excesiva de recursos, incremento de emisiones causadas por la movilidad de personas, congestión vehicular, incremento de residuos sólidos, entre otros.

La logística se define como la articulación de la provisión de servicios de infraestructura, la producción, la facilitación del movimiento, la distribución de bienes y la regulación de servicios e información a lo largo de la cadena global. Por ende, considera de manera integrada las necesidades de infraestructura, provisión de servicios, distribución y regulación de flujos de transporte y comerciales en un marco de objetivos de política

\footnotetext{
${ }^{1}$ Docente investigador. Carrera de logística y transporte. Universidad Politécnica Estatal del Carchi. Ecuador. daniel.beltran@upec.edu.ec

${ }^{2}$ Docente investigador. Carrera de logística y transporte. Universidad Politécnica Estatal del Carchi. Ecuador. gabriel.mafla@upec.edu.ec

${ }^{3}$ Docente investigador. Carrera de logística y transporte. Universidad Politécnica Estatal del Carchi. Ecuador. jonathan.mora@upec.edu.ec
} 
económica y organización territorial. Este enfoque brinda una utilización eficiente y sostenible de bienes de uso público y privado. (CEPAL, 2017)

La distribución urbana de mercancías es primordial para la dinámica económica de una ciudad y el bienestar de sus pobladores, debido a que influye directamente en la congestión, contaminación, consumo energético, seguridad vial y ocupación del espacio urbano. Los problemas de la distribución urbana de mercancías requieren soluciones integrales porque afectan a una gran diversidad de actores: los oferentes del servicio (transportistas y operadores logísticos) los demandantes (generadores de carga), las autoridades locales y los usuarios de la vía pública. (Antún, 2013)

La logística urbana se puede definir como el estudio de los movimientos de mercancías y de personas en la dinámica de la vida cotidiana de una ciudad. Para efectos del actual proyecto de investigación se ha dividido este concepto para, por un lado analizar el comportamiento individual de los movimientos de mercancías, y por el otro, el del movimiento de personas. Entenderemos como logística urbana al movimiento de mercancías que ocurre en una ciudad, dejando a un lado el estudio del movimiento de personas que lo entenderemos como movilidad urbana. Estas dos definiciones se han pensado pertinentes para el análisis de los movimientos de transporte generado por las mercancías (logística urbana), así como, los movimientos generados por el transporte de personas (movilidad urbana).

La logística urbana para su estudio tiene tres actores principales: los proveedores, receptores o clientes y la administración pública, existiendo entre estos actores principales un actor transversal que es el transporte, actor que interrelaciona y se encarga de la dinámica de las mercancías, que responde a una oferta y demanda, que en términos de logística urbana entenderemos como generación y atracción de viajes. (Antún, Lozano, Hernández, \& Hernández, 2005)

En el marco de trabajo del proyecto de investigación “Modelo de evaluación de sustentabilidad y de optimización de la movilidad y la logística urbanas, en la ciudad de Tulcán - Provincia del Carchi", se presentan los resultados obtenidos de la fase de diagnóstico, en donde se utilizan instrumentos de recolección de información, como registros, encuestas y entrevistas que sirvieron para obtener los datos de los actores involucrados en los movimientos de mercancías de la última milla. Adicionalmente, se utilizan datos no estructurados disponibles de acceso libre. Con esta información se procedió a realizar una descripción de la situación actual de los tres grupos principales de actores, como son los proveedores, receptores y administración pública, y se clarificó la dinámica de las interacciones existentes en la logística urbana de la ciudad de Tulcán, permitiendo determinar el comportamiento del movimiento de mercancías en la ciudad. En esta fase se recurrió a la teoría de grafos para entender las redes de distribución de cada proveedor y la relación con los clientes. La última sección tiene en su mayor parte contenido de opinión, reflejando la visión de los autores sobre el estado actual de la logística urbana en la ciudad de Tulcán. En este análisis se da una visión del desarrollo de los sistemas de distribución de mercancía que usan las empresas proveedoras, enfocados en la utilización de la capacidad de sus medios de transporte, así como en las rutas.

La investigación busca establecer modelos de evaluación y optimización de la movilidad y la logística urbana en la ciudad de Tulcán - Provincia del Carchi en Ecuador. La logística siendo considerada especialmente en los países industrializados como una herramienta vital para lograr competitividad a las empresas, regiones y países, no puede dejar de investigarse en el contexto nacional y regional. La falta de modelos de evaluación y de optimización en la zona 1 del Ecuador, específicamente en el cantón Tulcán, es un motivante para realizar el presente trabajo de investigación.

En la ciudad de Tulcán, las tecnologías de la información logística y nuevas metodologías tecnológicas actuales no son aplicadas de un modo adecuado y la falta de conocimiento sobre este tema tanto en la comunidad como 
en empresas del área, motivan a realizar este trabajo que apunta a sugerir metodologías de manera sustentable equilibrando las ganancias económicas, el bienestar social y buenas prácticas medioambientales, mientras se sugieren y analizan metodologías logísticas acorde a estándares actuales mundiales. Se pretende realizar una evaluación de los procedimientos de las empresas locales comparándolas con el estado del arte de vanguardia en las áreas de la logística y la movilidad urbana de una manera sustentable.

En el contexto local la problemática es el escaso conocimiento del desarrollo de las actividades en la movilidad y logística urbana por la falta de metodologías, buenas prácticas y estándares internacionales logísticos, dando como resultado costos excesivos de los productos y servicios y un bajo nivel de servicio al cliente.

La logística urbana estudia cómo las mercancías e información fluyen de forma eficiente, global y sostenible en un entorno urbano, superando el tiempo y la distancia. Las metodologías de competitividad económica bajo restricciones espaciales y de recursos, así como la sostenibilidad ambiental conllevan a aplicar los conceptos de logística al entorno urbano. Los sectores involucrados en la logística urbana son: Planificación, ingeniería y control, compras, producción y comercialización, distribución, clientes y administración local. Una optimización de la logística urbana puede contribuir a nuevas formas de organización, nuevos avances tecnológicos y mecanismos de control administrativos. Así como al establecimiento de una normativa al respecto que permita recaudación de impuestos, entrega de subvenciones y desarrollo de infraestructura. Para poder realizar el diagnóstico y respectivo análisis de la situación actual de la logística urbana de la ciudad de Tulcán tenemos los siguientes objetivos:

- Identificar los principales actores de la logística urbana de la ciudad de Tulcán y sus características.

- Caracterizar los movimientos de mercancías entre los diferentes actores.

- Realizar un análisis de la situación actual referente a la logística urbana de la ciudad de Tulcán.

\section{Metodología}

La investigación tuvo un paradigma principalmente de carácter cuantitativo, a la par también un enfoque cualitativo que permitió caracterizar el comportamiento de las variables como una base justificativa del por qué se presentan los resultados encontrados.

La investigación se desarrolló en tres fases, la primera de estructuración y validación de instrumentos de recolección de información como fueron entrevistas y encuestas. En una segunda instancia se realizó la zonificación y recolección de información, y con la información se procedió a la tercera parte que consistió en tabular, analizar y presentar los resultados obtenidos en la aplicación de las encuestas.

En la elaboración de encuestas se tomaron en cuenta los parámetros básicos para caracterizar los movimientos de mercancías en la ciudad de Tulcán, de esta manera se consideraron los factores de atracción de movimientos (demanda) y los factores de generación de movimientos (oferta), siendo los más importantes la zona, frecuencia de viajes, horario de viajes, tiempo de descarga, tal como se presenta en el cuadro 4 en la sección de anexos.

Para realizar la zonificación de la ciudad se tomaron en cuenta los parámetros más relevantes acordes a las características propias de cada sector y que pudieran ser determinantes en la generación y atracción de viajes. Factores tales como demografía, infraestructura, ocupación de suelo, actividad característica, se analizaron para determinar tres zonas de estudio, el norte de la ciudad (desde este punto se denominará zona 1) caracterizado por ser una zona residencial, el centro cuya actividad principal es el comercio (de aquí en adelante denominada zona 2) y el sur (identificada de aquí en adelante como zona 3) caracterizado por ser una zona residencial y de ubicación de centros de estudio superior. 
Con la información recolectada se procedió a determinar las frecuencias de viajes entre los puntos de origen y destino, utilizando grafos e histogramas de frecuencia para determinar los nodos de mayor generación y atracción de viajes, para de esta manera comprender los movimientos de mercancías en la ciudad de Tulcán.

\subsection{Instrumentos}

Los instrumentos utilizados en la investigación, entre otros que se pueden identificar como medios más eficientes de recolección de información durante el proceso, son la observación natural del investigador y la observación indirecta en forma de encuestas. Se utilizaron también encuestas de preferencias declaradas, en donde se plantearon situaciones hipotéticas y se registraron los resultados posibles, el instrumento utilizado se presenta en el cuadro 4.

Para realizar la zonificación se necesitó información referente a la población y demografía de la ciudad de Tulcán, teniendo como fuente oficial el registro del Instituto Nacional de Estadística y censos (INEC), existe una población de 86498 habitantes, en una superficie de 15 kilómetros cuadrados, teniendo una demografía de 5766,53 habitantes por kilómetro cuadrado. La ciudad se caracteriza por tener un crecimiento lineal de Norte a Sur, así pues las actividades comerciales se han concentrado a lo largo del tiempo en la parte central de la ciudad, no obstante en los sectores Norte y Sur se concentran las zonas residenciale (Instituto ecuatoriano de estadistica y censos, 2019)

Se realizó conteo y aforos de tráfico en los que se midieron los vehículos que pasan por determinados puntos de control o estaciones. Se utilizaron sistemas de información geográfica para generar mapas de rutas sobre las cuales se puede determinar e incorporar información dinámica del sistema.

Otro conjunto importante de necesidades del proyecto fueron los datos socioeconómicos de las personas que viven en el área de la investigación. Esta información se recolectó a través de encuestas basadas en el hogar, además de encuestas en lugares específicos como centros laborales y la vía pública.

Se utilizaron bases de datos disponibles en entidades relacionadas con la gobernanza, institucionalidad e investigación de sistemas de transporte, movilidad y logística urbana, sobre las cuales se realizó el análisis estadístico necesario y se determinó los parámetros relevantes para concluir sobre los resultados de la investigación.

\subsection{Técnicas de análisis y visualización de la información}

El aspecto clave de la investigación es la recolección de información confiable, basada en procedimientos estadísticos con objetivos descriptivo e inferencial, enfoques paramétricos, no paramétricos o bayesianos, y mediante análisis de tipo multivariante y funcional. De esta manera se pudo obtener la capacidad para interpretar y evaluar críticamente la información, los datos y fenómenos estocásticos que se encontraron en el proceso de investigación y capacidad para comunicar las evidencias, causas y conclusiones relevantes al problema de estudio. Se realizó el ajuste de los datos con modelos de regresión lineal.

Los procesos de análisis de los modelos de datos masivos y la visualización dinámica de los datos y de los resultados, se realizaron mediante la programación de herramientas informáticas especializadas en ciencia de datos que permiten un tratamiento sencillo y eficaz de los gráficos de control de calidad de la información como R, Matlab y Python.

\subsection{Tipo de investigación}

El análisis contempló una fase de investigación exploratoria con el hallazgo de datos secundarios que se recolectaron de investigaciones recientes generadas alrededor del mundo acerca de las temáticas de movilidad 
urbana y logística urbana, luego se contempló una etapa de recolección de datos primarios para generar investigación causal descriptiva y el respectivo análisis de datos cuantitativo de las variables establecidas como necesarias.

\section{Resultados}

Para realizar el diagnóstico situacional de la logística urbana en la ciudad de Tulcán, fue necesario caracterizar y conocer los actores involucrados en el movimiento de mercancías en el area urbana, determinar la dinamica existente entre estos actores y las caracteristicas de infraestructura y normativa existentes en el espacio motivo de la investigación. Para conocer a los actores y establecer sus ubicaciones se procedió al levantamiento de información mediante la aplicación de encuestas en campo, de donde se obtuvieronn los siguientes resultados.

\subsection{Proveedores}

Para la caracterización de este sector se hizo una subdivisión que se basó en el tipo de movimiento generado por los proveedores. Quienes generan un movimiento alto de mercancías en el caso de la ciudad de Tulcán son quienes distribuyen productos de consumo masivo, por otra parte quienes proveen productos comerciales (ropa, calzado, lencería) generan un movimiento bajo de mercancías. Para efecto del estudio no se tomaron en cuenta a los proveedores de productos comerciales, ya que los movimientos que generan son muy bajos con una frecuencia de 1 aprovisionamiento mensual en el caso más significativo.

Para os proveedores de productos de consumo masivo se definieron dos categorías en el tipo de aprovisionamiento, la entrega en el punto de consumo (realizada por lo proveedores) y el aprovisionamiento en el centro de distribución (realizada por los consumidores). Cabe indicar que para el estudio se tomaron en consideración aquellos proveedores que generan la mayor cantidad de movimientos en el entorno urbano, debido al impacto generado por estos en el flujo vehícular, emisiones al ambiente, generacion de ruido y demás impactos que se producen por el uso de medios de transporte motorizado.

Para una mejor comprensión de esta clasificación los resultados de este levantamiento de información a continuación se presenta el cuadro 1. 
Cuadro 1

Clasificación de proveedores

\begin{tabular}{|c|c|c|c|}
\hline $\begin{array}{l}\text { Movimiento de } \\
\text { mercancías por } \\
\text { mensual }\end{array}$ & Tipo de mercancías & Tipo de aprovisionamiento & Proveedor \\
\hline \multirow{32}{*}{15} & \multirow{32}{*}{$\begin{array}{c}\text { Productos de consumo masivo (víveres, } \\
\text { productos de aseo, productos para el } \\
\text { hogar) }\end{array}$} & \multirow{16}{*}{$\begin{array}{l}\text { Entrega realizada por el proveedor en } \\
\text { punto de consumo }\end{array}$} & ARCA CONTINENTAL \\
\hline & & & CARLOS ARIAS \\
\hline & & & COILE SA \\
\hline & & & MULTIPRODUCTOS \\
\hline & & & PASTEURIZADORA QUITO \\
\hline & & & ORIENTAL \\
\hline & & & PINGÜINO \\
\hline & & & PARMALAT \\
\hline & & & TONICORP \\
\hline & & & CERVECERÍA NACIONAL \\
\hline & & & COLOMBINA \\
\hline & & & CONFITECA \\
\hline & & & QUALA \\
\hline & & & UNIVERSAL \\
\hline & & & CORDIALSA \\
\hline & & & TESALIA \\
\hline & & \multirow{16}{*}{$\begin{array}{l}\text { Aprovisionamiento realizado por el } \\
\text { consumidor en centro de distribución }\end{array}$} & MERCADO SAN MIGUEL \\
\hline & & & MERCADO DEL SUR \\
\hline & & & MERCADO CENTRAL \\
\hline & & & SUPERMAXI \\
\hline & & & COMERCIAL CADENA \\
\hline & & & ABASTOS D'GIOVYS \\
\hline & & & HUEVOS NORMI \\
\hline & & & ROSA ORDOÑES \\
\hline & & & BODEGA POPULAR TABACUNDO \\
\hline & & & SUPER EXPRESS \\
\hline & & & COMERCIAL SAN SEBASTIÁN \\
\hline & & & BODEGA CARLOS \\
\hline & & & BODEGA AGROCOMODITIES \\
\hline & & & COMERCIAL CORDOVA \\
\hline & & & BODEGAS ANITA'S \\
\hline & & & COMERCIAL CASTILLO \\
\hline 1 & $\begin{array}{l}\text { Productos comerciales (ropa, zapatos, } \\
\text { lencería) }\end{array}$ & $\begin{array}{l}\text { Aprovisionamiento realizado por el } \\
\text { consumidor en centro de distribución }\end{array}$ & VARIOS \\
\hline \multicolumn{3}{|c|}{ Total proveedores que entregan en punto de consumo } & 16 \\
\hline \multicolumn{3}{|c|}{ Total centros de distribución } & 16 \\
\hline
\end{tabular}

Fuente: Elaboración propia 


\subsection{Receptores o consumidores}

Los consumidores son la principal fuente de atracción de los movimientos de mercancías y están distribuidos en toda la ciudad. Estos tienen puntos de consumo en común que son las tiendas de abarrotes ubicadas en los diferentes barrios. Para levantar la información de estos nodos comunes de consumo se realizó en primer lugar una zonificación de la ciudad determinando tres zonas: norte denominada para el estudio zona 1 (límite norte de la ciudad hasta parque Ayora), el centro denominada zona 2 (desde el parque Ayora hasta el terminal terrestre de la ciudad) y sur denominada zona 3 (desde el terminal terrestre hasta el límite sur de la ciudad), en donde, se identificaron 138 tiendas de abasto, que para efecto de la investigación son los nodos de atracción de viajes y de acuerdo a la zona tienen la siguiente distribución de acuerdo al gráfico 1.

Gráfico 1

Distribución de puntos de consumo

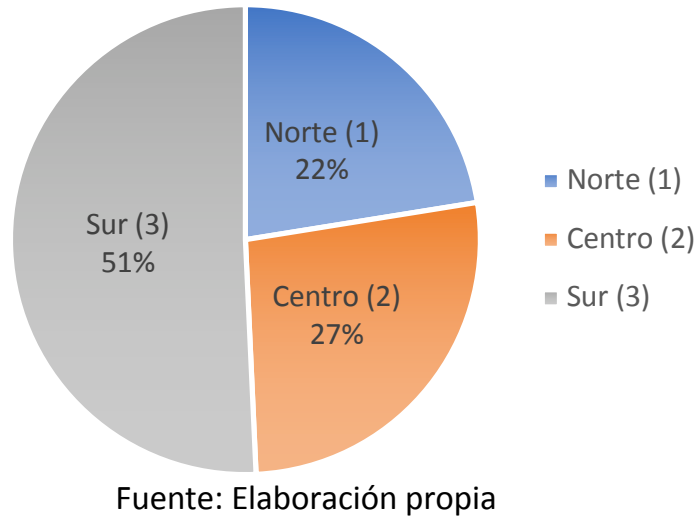

Al revisar la información presentada en el gráfico 1 sobre los puntos de consumo (tiendas de abarrotes), podemos identificar que el $51 \%$ están ubicados en el sector sur de la ciudad, esto se debe principalmente a las actividades típicas de cada zona. En la zona centro se encuentra el sector comercial, mientras que el norte es un sector residencial al igual que el sector sur. La diferencia entre las zonas norte y sur en el número de puntos de consumo, se debe a que en el sector sur de la ciudad se encuentran ubicados dos centros de educación superior que son un factor determinante en lo que respecta a la atracción de movimientos de mercancías de consumo masivo por su densidad poblacional.

\subsection{Administración pública}

La administración pública es un factor importante en la logística urbana debido a que es quien regula y brinda las facilidades para que exista una adecuada interacción entre los otros actores generadores y atractores de movimientos de mercancías.

En el levantamiento de información se determinaron varios parámetros pertinentes a la administración pública como son: infraestructura, políticas de uso, regulaciones, estos aspectos se resumen en el cuadro 2 para un mejor entendimiento.

Cuadro 2

Administración pública

\begin{tabular}{|l|l|l|}
\hline \multirow{4}{*}{ Infraestructura } & Vías de primer orden para circulación vehicular & $\begin{array}{l}\text { Existen vías de primer orden en toda la } \\
\text { ciudad para la circulación vehicular }\end{array}$ \\
\cline { 2 - 3 } & Carril exclusivo para circulación de vehículos de carga & $\begin{array}{l}\text { No existen carriles exclusivos para } \\
\text { vehículos de carga }\end{array}$ \\
\cline { 2 - 3 } & Zonas exclusivas de carga/descarga & $\begin{array}{l}\text { No existen zonas de carga y descarga } \\
\text { en los lugares en donde se realizan } \\
\text { estas actividades }\end{array}$ \\
\hline
\end{tabular}




\begin{tabular}{|l|l|l|}
\hline \multirow{4}{*}{ Normas regulatorias } & Restricción zonal de circulación de vehículos de carga & $\begin{array}{l}\text { Se restringe la circulación de vehículos } \\
\text { grandes en determinadas zonas de la } \\
\text { ciudad }\end{array}$ \\
\cline { 2 - 3 } & Horarios para circulación de vehículos de carga & $\begin{array}{l}\text { No existe restricción horaria para la } \\
\text { circulación de vehículos de carga }\end{array}$ \\
\cline { 2 - 3 } & Restricción de tipos de vehículos de carga & $\begin{array}{l}\text { Existe restricción de vehículos grandes } \\
\text { de carga }\end{array}$ \\
\hline
\end{tabular}

Fuente: Elaboración propia

La regulación de la circulación vehicular en cuanto a la movilización de vehículos de carga en la ciudad de Tulcán no se encuentra actualizada. Se puede presumir que no se le da la relevancia esperada ya que, al ser una ciudad con una población relativamente pequeña, aun no se han experimentado problemas graves de congestión vehicular causados por el movimiento de mercancías.

\subsection{Distribución de mercancías}

Las empresas que distribuyen productos de consumo masivo tienen sus particularidades, de acuerdo al producto que comercializan. La información que arroja el estudio sobre las frecuencias de entrega, la cantidad de vehículos de distribución y las zonas en donde se realizan estas entregas se presenta en el cuadro 3.

\section{Cuadro 3}

Número de vehículos distribuidores por zona

\begin{tabular}{|c|c|c|c|c|c|c|c|c|c|c|c|c|c|c|c|c|c|c|}
\hline Dia & \multicolumn{3}{|c|}{ Lunes } & \multicolumn{3}{|c|}{ Martes } & \multicolumn{3}{|c|}{ Miércoles } & \multicolumn{3}{|c|}{ Jueves } & \multicolumn{3}{|c|}{ Viernes } & \multicolumn{3}{|c|}{ Sábado } \\
\hline Zona & $1^{*}$ & $2 * *$ & $3 * * *$ & 1 & 2 & 3 & 1 & 2 & 3 & 1 & 2 & 3 & 1 & 2 & 3 & 1 & 2 & 3 \\
\hline Arca & 2 & 2 & 1 & & & & & & & & & & & & & 1 & 1 & 1 \\
\hline Carlos arias & 2 & 2 & 1 & & & & & & & & & & 1 & 1 & 1 & & & \\
\hline Coile s.a. & 1 & 1 & 1 & & & & & & & & & & & & & 1 & 1 & 1 \\
\hline Multiproductos & 2 & 2 & 2 & & & & & & & & & & 2 & 2 & 2 & & & \\
\hline Pasteurizadora quito & 2 & & 2 & 2 & & 2 & 2 & & 2 & 2 & & 2 & 2 & & 2 & 2 & & 2 \\
\hline Oriental & 2 & 2 & 2 & 2 & 2 & 2 & 2 & 2 & 2 & 2 & 2 & 2 & 2 & 2 & 2 & 2 & 2 & 2 \\
\hline Pingüino & 1 & & 1 & 1 & & 1 & 1 & & 1 & 1 & & 1 & 1 & & 1 & 1 & & 1 \\
\hline Parmalat & 1 & & 1 & & & & & & & & & & & & & 1 & & 1 \\
\hline Tonicorp & & & & 1 & 1 & 1 & & & & & & & 1 & 1 & 1 & & & \\
\hline Cerveceria nacional & 1 & 2 & 1 & & & & & & & & & & & & & 1 & 2 & 1 \\
\hline Colombina & 2 & 2 & 1 & & & & & & & & & & 2 & 2 & 1 & & & \\
\hline Confiteca & 2 & 2 & 2 & 2 & 2 & 2 & 2 & 2 & 2 & 2 & 2 & 2 & 2 & 2 & 2 & 2 & 2 & 2 \\
\hline Quala & 1 & 1 & 1 & & & & & & & & & & & & & 1 & 1 & 1 \\
\hline Universal & 1 & & & & & & & 1 & 1 & 1 & & & & & & & 1 & 1 \\
\hline Cordialsa & 1 & & & 1 & & & & 1 & & & 1 & & & & & & & 1 \\
\hline Tesalia & & 1 & & & & & & 1 & & 1 & & & & & 2 & & & \\
\hline Total por zona & 21 & 17 & 16 & 9 & 5 & 8 & 7 & 7 & 8 & 9 & 5 & 7 & 13 & 10 & 14 & 12 & 10 & 14 \\
\hline Total por día & & 54 & & & 22 & & & 22 & & & 21 & & & 37 & & & 36 & \\
\hline
\end{tabular}

* Zona Norte denominada para el estudio zona 1 (límite Norte de la ciudad hasta parque Ayora),

** Centro de la ciudad denominada zona 2 (desde el parque Ayora hasta el terminal terrestre de la ciudad)

*** Sur denominada zona 3 (desde el terminal terrestre hasta el límite Sur de la ciudad)

Fuente: Elaboración propia

Al analizar esta información podemos determinar que los días en los que se realiza un mayor movimiento de mercancías son los lunes, viernes y sábado, en los que todas las empresas realizan entregas de productos. Esta dinámica es característica en casi todas las empresas que distribuyen productos de consumo masivo, debido a 
que cada punto de consumo se aprovisiona al inicio de la semana (lunes) para la demanda semanal y el día viernes dependiendo del consumo en el transcurso de estos días se reaprovisiona para terminar la semana, podemos observar esta información en el grafico 2.

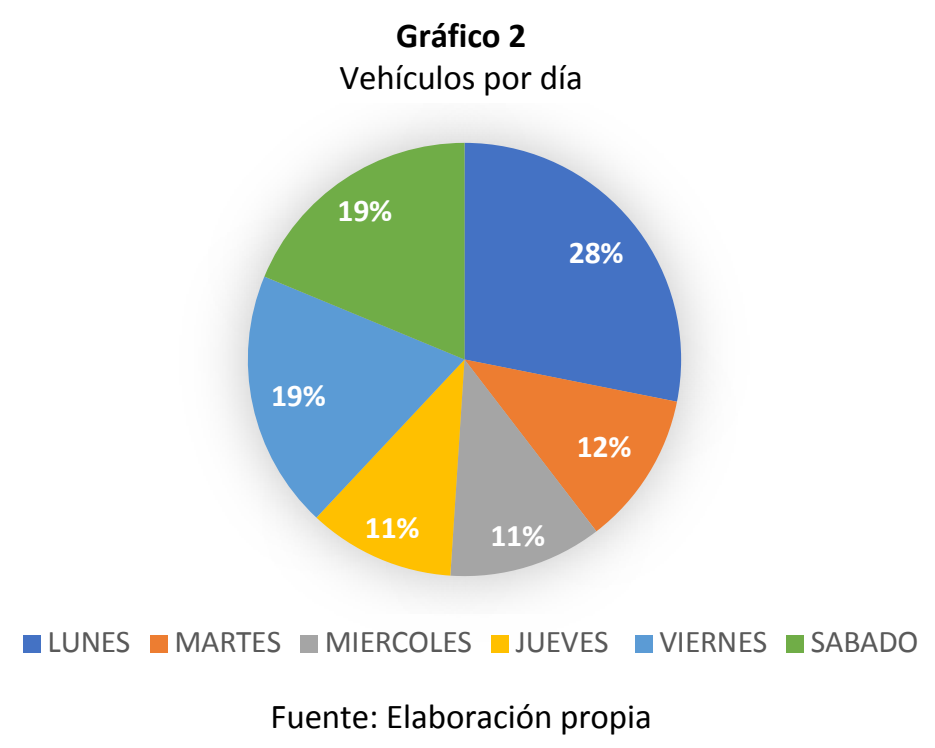

Al revisar la información resumida en el cuadro 3 se observa que la zona de la ciudad que atrae más movimientos de mercancías es la Norte con un total de 71 vehículos semanalmente, que representan el $37 \%$ de viajes, seguida por la zona Sur con un total de 66 vehículos, es decir el 35\% de movimientos de carga y, por último, la zona centro con un total de 54 vehículos distribuidores, siendo el $28 \%$ en el último caso. Podemos identificar que los movimientos de cargas mayoritariamente se realizan en el norte y el sur debido a que estas son zonas residenciales, a diferencia de la zona centro que es una zona comercial.

\subsection{Análisis por distribuidor}

El distribuidor de la empresa Tesalia, en la ciudad de Tulcán, para realizar la entrega de sus productos a las tiendas de la localidad utiliza 4 vehículos de 3 toneladas, los cuales realizan el siguiente recorrido: un vehículo recorre la zona Norte, otro vehículo la zona centro y 2 vehículos la zona Sur. En el cuadro 3 se puede observar que el centro es el sector de menor cantidad de movimientos y que en el Norte y el Sur se realiza la mayor cantidad de pedidos, por lo que se realizan más movimientos en estos sectores de la ciudad. Los automóviles que trasladan la carga no son utilizados en su totalidad, la mayor parte de sus pedidos apenas corresponde a la mitad (50\%) de la capacidad de los automóviles.

COILE realiza las entregas de sus productos mediante un único vehículo que recorre las tres zonas de la ciudad los días lunes y sábado, ocupando su capacidad, en el mejor de los casos, en un $60 \%$.

Cervecería Nacional es una de las empresas más grandes del país ya que distribuye a todas las provincias de Ecuador, una de ellas es Tulcán. El centro de distribución principal se encuentra ubicado en la provincia de Guayaquil. Para la ciudad objeto de estudio la empresa realiza el abastecimiento a 107 tiendas con 4 camiones, los cuales realizan sus entregas los días lunes y sábado, dos vehículos hacen la entrega en la zona centro, uno a la zona Norte y uno a la zona Sur, esto se debe a que la mayor cantidad de licorerías y bares se encuentran ubicados en la zona central de la ciudad.

La empresa Pasteurizadora Quito, productora y distribuidora de productos lácteos para los diferentes sectores de la ciudad y alrededores, cuenta con cuatro vehículos cubiertos y refrigerados con una capacidad de 3 
toneladas cada uno. Los productos se distribuyen en toda la ciudad de Tulcán directamente a los clientes. En el norte abastece a 46 tiendas, en el centro abastece a 27 tiendas y en el sur a 50 tiendas.

Parmalat, en la provincia del Carch,i realiza su distribución los lunes y sábados, para lo que utiliza 2 vehículos con 3 toneladas de capacidad. Según los datos obtenidos, la empresa abastece en el sector Norte a 23 puntos locales, en el sector centro a 10 puntos de venta y en el sector Sur a 54.

Distribuidora Carlos Arias lleva sus productos a la ciudad de Tulcán los días lunes, miércoles y viernes a 101 tiendas, la empresa tiene el siguiente sistema de distribución: día lunes zona 3, miércoles zona 2 y viernes zona 1. Ocupan 2 camiones de 5 toneladas y dos camiones de 7 toneladas. En la zona 3, realizan la distribución a 49 tiendas, en la zona 2 se encuentran 15 tiendas y en la zona 1 se encuentran 37 tiendas.

Pingüino tiene su distribuidor en Tulcán para lo cual utiliza 2 camiones con refrigeración que recorren la ciudad entregando los productos y de esta forma satisface a las necesidades de los clientes. La mayoría de las tiendas que ofrecen esta marca cuentan con una gran variedad de productos.

El distribuidor Confiteca reparte sus productos a la ciudad de Tulcán desde hace varios años, todo el portafolio de productos es repartido por un camión que, dependiendo de los pedidos, planifica su ruta para cumplir con la demanda de las tiendas de toda la ciudad.

La empresa Colombina en Tulcán se encarga de realizar el recorrido de entrega los días lunes y viernes repartiendo a un total de 113 tiendas ubicadas en la zona urbana, utiliza 5 vehículos que llevan un peso total aproximado de 265 kilos de producto variado para la entrega de los cuales tienen diferentes rutas establecidas, 2 son destinados al sector norte con 30 tiendas, 2 al sector sur con 66 tiendas y 1 al centro con 17 tiendas.

La empresa la Universal para facilitar la distribución se ha dividido en tres rutas para realizar la entrega de sus productos a todas las tiendas de Tulcán. Entrega sus productos mediante tres camiones cada uno para cada zona, en la zona 1 los días de recorrido son los lunes y jueves, en la zona 2 lo realizan los miércoles y sábado y la zona 3 los días miércoles y sábado respectivamente, visitando un total de 128 tiendas en la ciudad de Tulcán.

Las empresas mencionadas realizan la distribución de la mayor cantidad de productos de consumo masivo en la ciudad de Tulcán con una frecuencia establecida. Existe también una pequeña cantidad de productos que se distribuyen como plásticos y productos que tienen un índice bajo de rotación. Debido a esto, los movimientos de carga no son representativos y sería importante tomarlos en cuenta en un estudio que cuente con aplicación de tecnología adecuada para controlar todos los movimientos existentes de carga. No obstante, en el gráfico 4 se presenta un resumen de los movimientos de carga generados por día, en donde podemos observar que la mayor movilización de vehículos ocurre los lunes, así también, podemos identificar que en este día los mayor cantidad de movimientos son atraídos por la zona 1. También podemos identificar que los viernes y sábado son los que le siguen en cuanto a cantidad de vehículos de carga movilizados, teniendo los días faltantes de la semana una movilización baja de vehículos de distribución. Ninguna de estas empresas realiza entregas a sus puntos de consumo el día domingo.

A partir de la información presentada en los cuadros anteriores, podemos realizar un grafo de circulación vehicular de los tres días con mayor afluencia de vehículos distribuidores de productos de consumo masivo en la ciudad de Tulcán, lunes, viernes y sábado, en los cuales fácilmente se puede identificar las zonas en las que se podría encontrar problemas de congestión. Cabe indicar que debido a que la densidad poblacional de la ciudad de Tulcán es baja, 5766,53 habitantes por kilómetro cuadrado, hasta el momento no se han presentado problemas graves de congestión, pero siempre es importante planificar a futuro para prevenir este y otros problemas que se presentarían cuando la demanda vehicular sobrepase la capacidad de la infraestructura con la que actualmente cuenta la ciudad. 
Gráfico 3

Vehículos por zona

Viajes generados por distribución de productos de consumo masivo

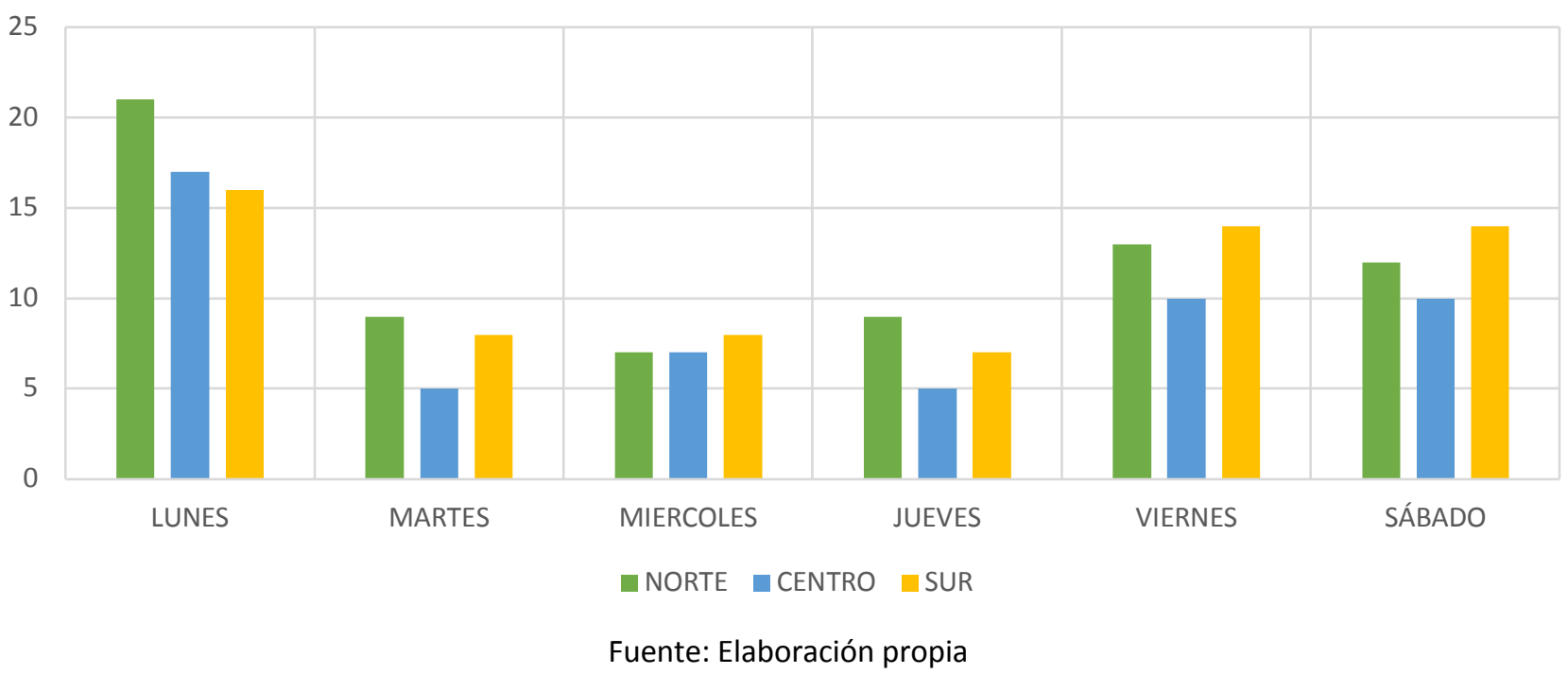

Se puede observar en el gráfico 4 la representación de la distribución de productos de consumo masivo los días lunes que todas las empresas realizan envíos de mercancía este día, a excepción de la empresa Tonicorp, además, podemos observar que la zona de mayor atracción de viajes para mercancía es la 1 , dato que constituye un indicio para la tooma decisiones a futuro en cuanto a políticas de circulación o infraestructura, decisiones que permitan evitar problemas de congestión vehicular en el caso de un crecimiento demográfico, mismo que conlleva a un crecimiento vehicular.

En el gráfico 4 observamos que la atracción de viajes en las tres zonas de la ciudad es menor que el día lunes. Las zonas que motivan más viajes son la zona 2 y la zona 3. En el caso del día viernes la zona que atrae más viajes es la 3 , además, podemos observar que el $44 \%$ de empresas no realiza envíos a ninguna zona en este día, a diferencia del día lunes en donde solo una empresa no realiza envíos.

El gráfico 6 nos muestra la dinámica de movimiento de mercancías que se genera el día sábado en la ciudad de Tulcán, podemos identificar que al igual que los otros días, las zonas que atraen más viajes son la zona 1 y 2 , siendo en este día la zona 3 la que atrae más viajes.

Al caracterizar los movimientos de productos de consumo masivo se pueden identificar cuáles son las zonas que atraen la mayor cantidad de viajes en la ciudad de Tulcán. De igual manera podemos definir los días en que se producen más viajes, siendo estos los críticos en la posible generación de congestión vehicular a causa de la mayor afluencia de vehículos de distribución. Esto nos permite planificar a futuro en caso de un incremento demográfico, dependiendo de este incremento y de los recursos disponibles, esta planificación podría consistir en políticas de circulación vehicular, como pueden ser horarios y rutas exclusivas, o en el caso de existir mayor disponibilidad de recursos incluso el mejoramiento de la infraestructura urbana para la circulación vehicular.

Para comprender el grafo se presenta la nomenclatura utilizada para identificar a cada una de las empresas que distribuyen los productos en la ciudad de Tulcán. 
Cuadro 1

Nomenclaturas de empresas/sector de la ciudad

Empresa/sector de la ciudad

Nomenclatura

\begin{tabular}{ll}
\hline ARCA CONTINENTAL & $\mathrm{A}$ \\
\hline CARLOS ARIAS & $\mathrm{B}$ \\
\hline COILE SA & $\mathrm{C}$ \\
\hline MULTIPRODUCTOS & $\mathrm{D}$ \\
\hline PASTEURIZADORA QUITO & $\mathrm{E}$ \\
\hline ORIENTAL & $\mathrm{F}$ \\
\hline PINGÜINO & $\mathrm{G}$ \\
\hline PARMALAT & $\mathrm{H}$ \\
\hline TONICORP & $\mathrm{I}$ \\
\hline CERVECERÍA NACIONAL & $\mathrm{J}$ \\
\hline COLOMBINA & $\mathrm{K}$ \\
\hline CONFITECA & $\mathrm{L}$ \\
\hline QUALA & $\mathrm{M}$ \\
\hline UNIVERSAL & $\mathrm{N}$ \\
\hline CORDIALSA & $\mathrm{O}$ \\
\hline TESALIA & $\mathrm{P}$ \\
\hline ZONA NORTE & 1 \\
\hline ZONA CENTRO & 2 \\
\hline ZONA SUR & 3 \\
\hline
\end{tabular}




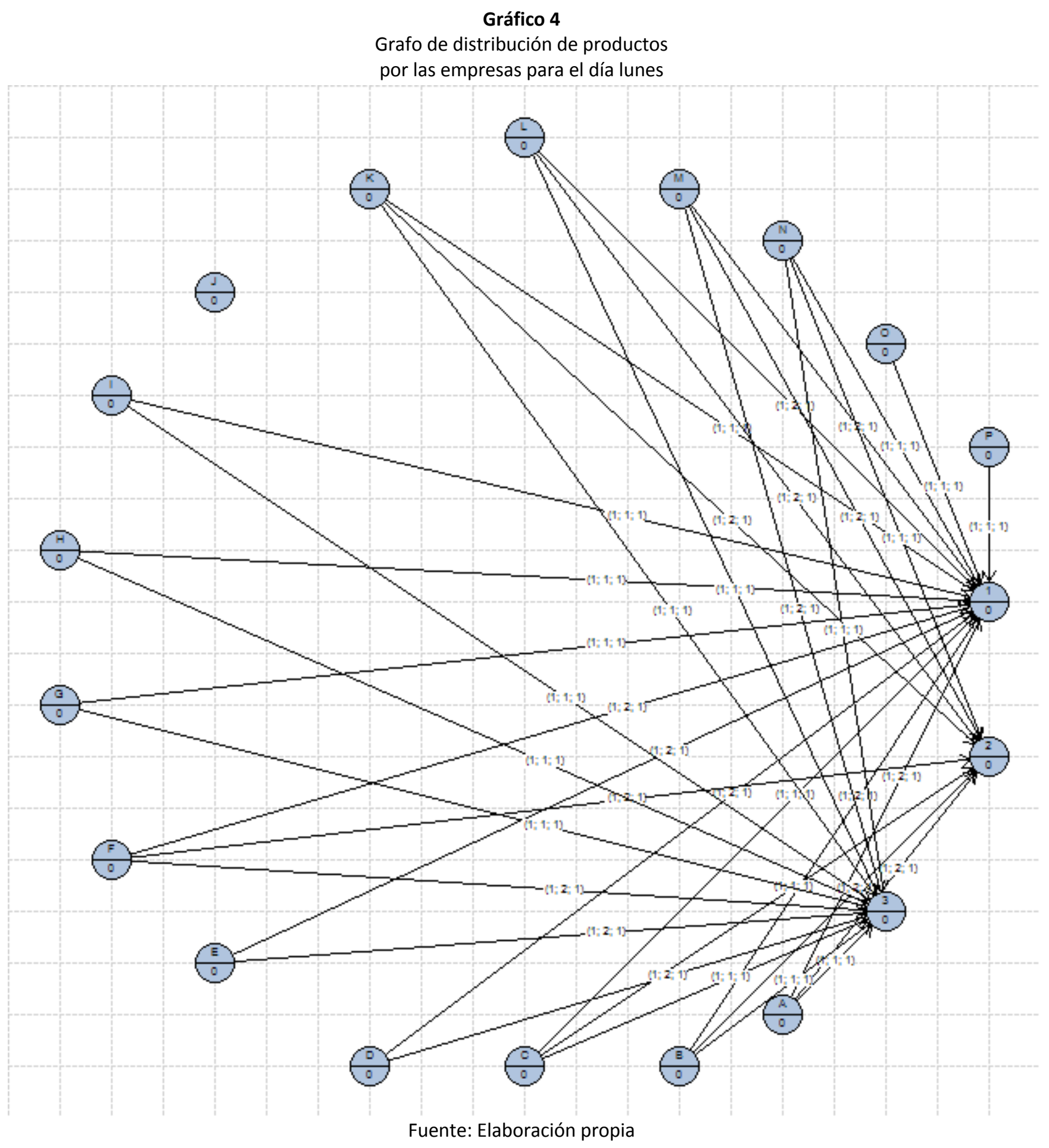

Los números presentados en las líneas (arcos) de los grafos son datos de distancia recorrida y tipo de almacén atendido, que para efecto del presente estudio son irrelevantes. 
Gráfico 5

Grafo de distribución de productos del día viernes

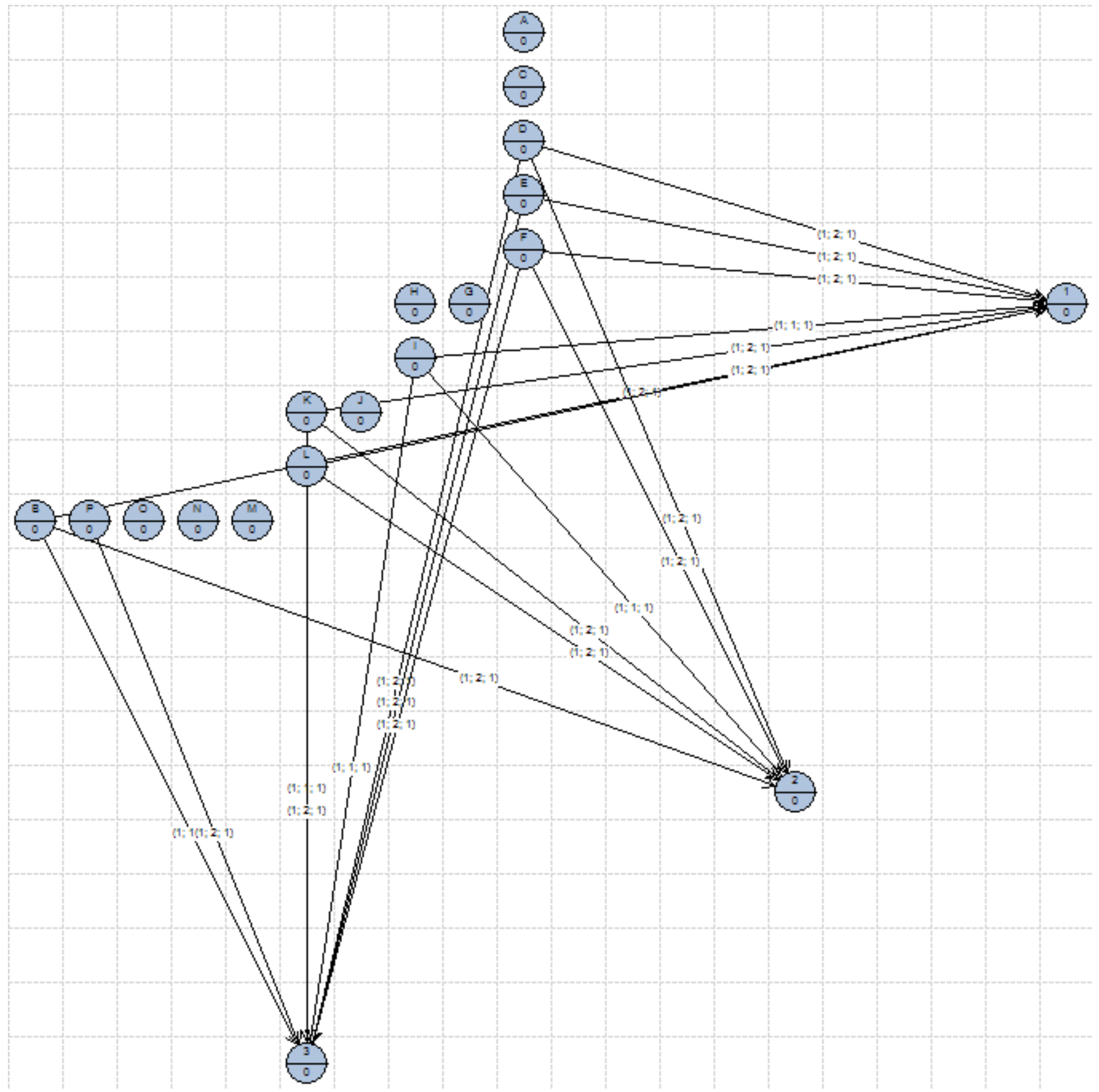

Fuente: Elaboración propia 


\section{Gráfico 6}

Grafo de distribución del día sábado

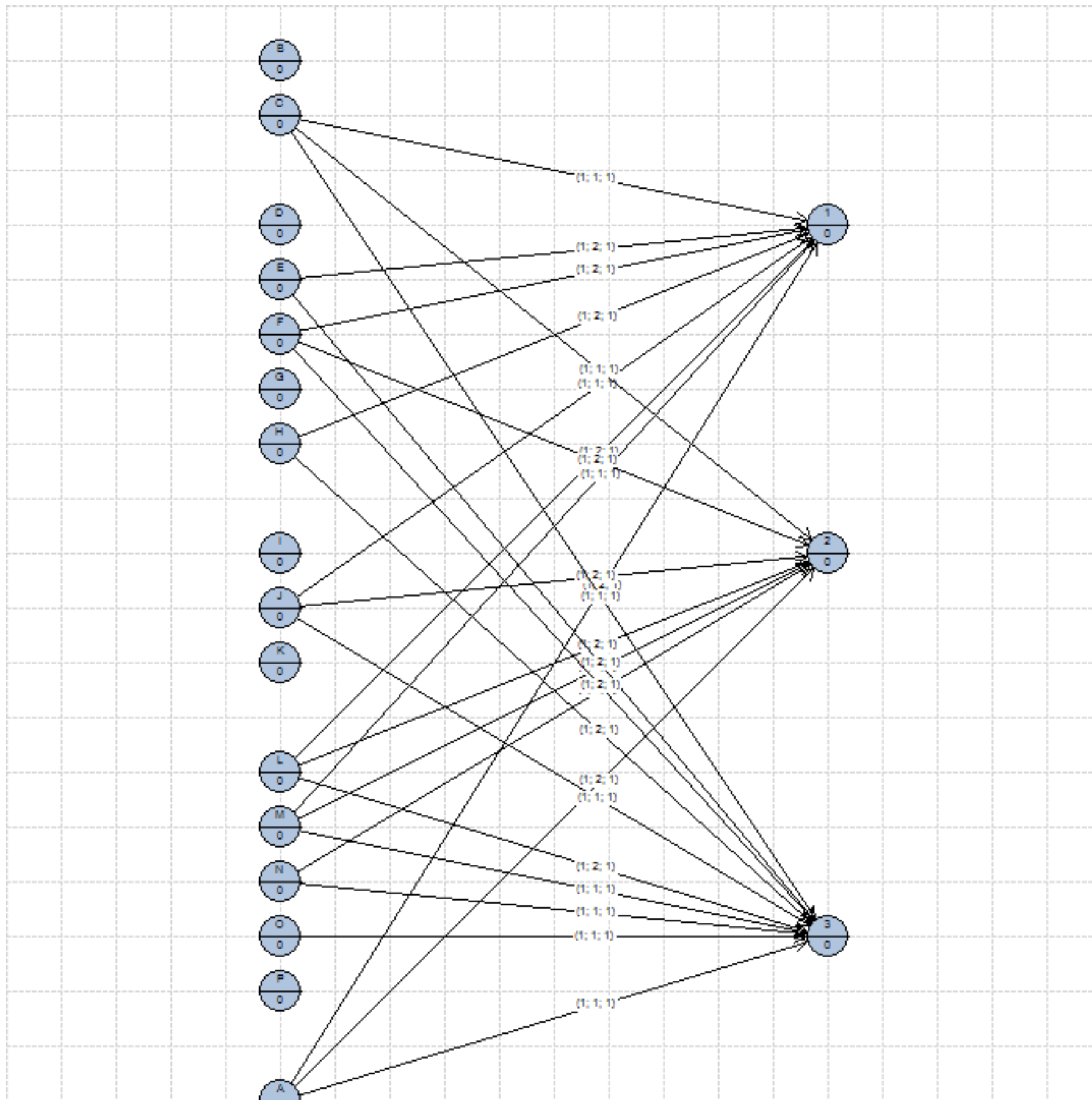

Fuente: Elaboración propia

\section{Conclusiones}

La logística urbana de la ciudad de Tulcán se ecuentra principalmente influída por la oferta y la demanda de productos de consumo masivo, no obstante, los productos que generan comercio (ropa, calzado, electrodomesticos) también producen afluencia de vehiculos de carga, pero estos no representan una cantidad significativa que afecte el flujo vehicular de la ciudad. Por lo tanto, se identifica que en la ciudad de Tulcán, los 
principales actores de los movimientos de carga son los puntos de consumo o demanda (tiendas y micromercados) y los distribuidores de productos de consumo masivo u oferta. Estos dos actores se encuentran regulados por un actor que es la administración pública, que se encarga de la normativa e infraestructura urbana. La ciudad de Tulcán cuenta con varios puntos de consumo que son centros de atracción de viajes de mercancías, asi tenemos 3 mercados, 2 supermercados y 138 tiendas y micromercados aproximadamente, siendo estas últimas las que atraen la mayor cantidad de viajes de provedores. Los principales proveedores son 16, quienes se encargan de la distribucion de productos a los diferentes puntos de consumo.

La ciudad de Tulcán se encuentra claramente zonificada en tres sectores, el sector norte que se caracteriza por ser un lugar residencial, el sector centro que es un sector comercial y el sector sur en el que se encuentran centros de educación superior y residencias. Las zonas norte y sur, son las zonas que atraen la mayor cantidad de viajes de los distribuidores de productos de consumo masivo, esto responde a la realidad de que las dos zonas son residenciales y presentan mayor demanda de estos productos, así pues, el 37\% de los viajes de mercancías son atraídos por la zona norte, el $35 \%$ por la zona sur y el $28 \%$ por la zona centro. Los días que mayor afluencia de vehículos de carga existe son los días lunes representando el $28 \%$ de los viajes que se realizan en la semana y los días viernes y sábado cada uno con un $18 \%$ del total de viajes que se realizan en la semana. Podemos identificar claramente que los días en los que se pueden presentar problemas de congestión vehicular a causa de los movimientos de mercancía son los días lunes, viernes y sábado en las zonas norte y sur de la ciudad.

Debido a que la densidad poblacional de la ciudad de Tulcán es relativamente baja, en la actualidad no existen problemas en lo que se refiere a congestión vehicular, no obstante, el estudio realizado permite planificar a futuro, para evitar problemas relacionados con el movimiento de cargas y las posibles repercusiones que esto podría conllevar. Podemos identificar que, al no existir la necesidad de contrarrestar una congestión vehicular por el movimiento de cargas, tampoco existe una norma regulatoria para las mismas, como puede ser un horario de movilización de vehículos de carga, zonas específicas para carga y descarga de productos o rutas específicas para este propósito. Las zonas que podrían tornarse críticas por el movimiento de mercancías están identificadas claramente: la 1 y la 3. Al determiarse que los días de mayor tráfico son los lunes, viernes y sábado, este sería un punto de partida para realizar una regulación por parte de la administración pública de la ciudad de Tulcán.

\section{Referencias bibliográficas}

Antún, J. P. (2013). Distribución Urbana de Mercancías: Estrategias con Centros Logísticos . Banco interamericano de desarrollo, 2-5.

Antún, J. P., Lozano, A., Hernández, J. C., \& Hernández, R. (2005). Logística de distribución física a minoristas. México: Universidad Nacional Autónoma de México.

Beltrán D. (2018). Logística urbana y su desarrollo hasta la actualidad. Revista visión empresarial. Volumen 9. Páginas $182-189$.

CEPAL. (2017). Políticas de logística y movilidad para el desarrollo sostenible y la integración regional. Recursos naturales e infraestructura, 14-19.

Instituto Ecuatoriano de Estadística y Censos. (6 de Mayo de 2019). INEC. Obtenido de https://www.ecuadorencifras.gob.ec/censo-de-poblacion-y-vivienda/

Ragas P. (2018). Logística urbana: Manual para operadores logísticos y administraciones públicas. 1ạ edición. Biblioteca de logística. Barcelona, España. 


\section{Anexos}

\section{Cuadro 4}

Modelo de encuesta aplicada

Saludos cordiales, la presente entrevista es una herramienta de recolección de información que servirá a la Carrera de Logística y Transporte de la UPEC, a determinar la situación actual de la logística urbana en la ciudad de Tulcán, dimensión que está siendo estudiada en el marco del proyecto de investigación denominado "Modelo de evaluación de sustentabilidad y de optimización de la movilidad y la logística urbanas, en la ciudad de Tulcán - Provincia del Carchi".

La información que usted nos proporcione servirá diagnosticar la situación actual y emitir propuestas de mejora en caso de ser necesarias, en lo que respecta a la logística urbana de la ciudad de Tulcán.

ACUERDO DE CONFIDENCIALIDAD: La Carrera de logística y Transporte se compromete a utilizar la información proporcionada en esta entrevista con fines única y exclusivamente investigativos, asi como la confidencialidad de la información, comprometiéndose a no divulgar información que sea de uso exclusivo de la empresa y que pueda comprometer sus intereses.

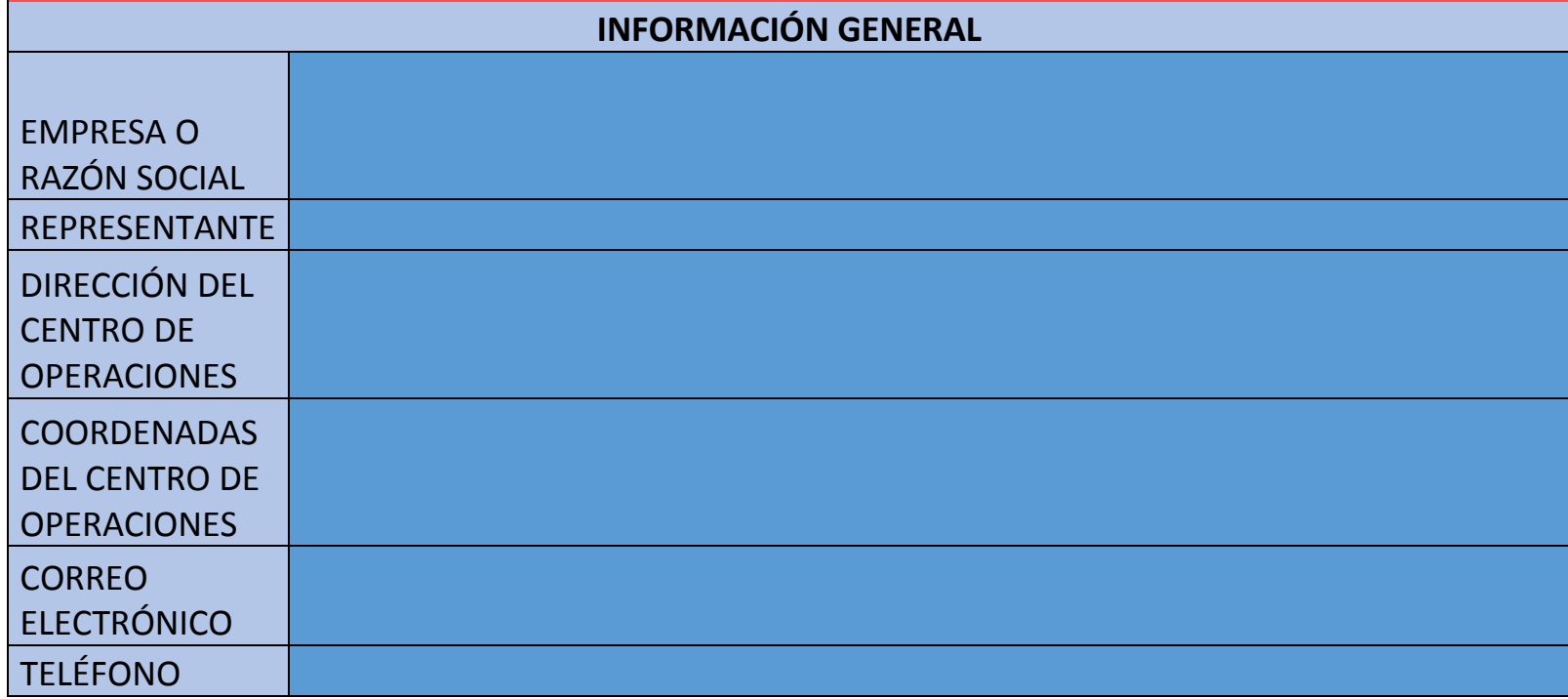

INFORMACIÓN DE LA ESTRUCTURA EMPRESARIAL

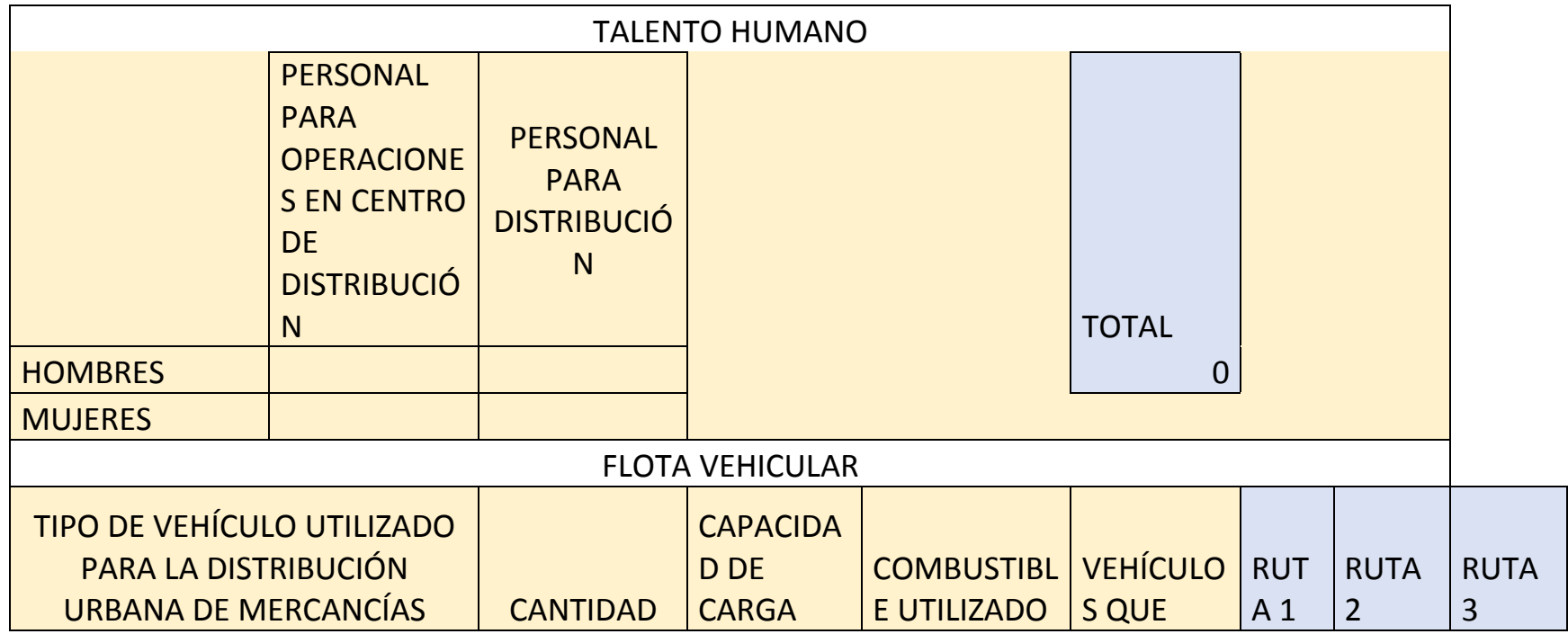




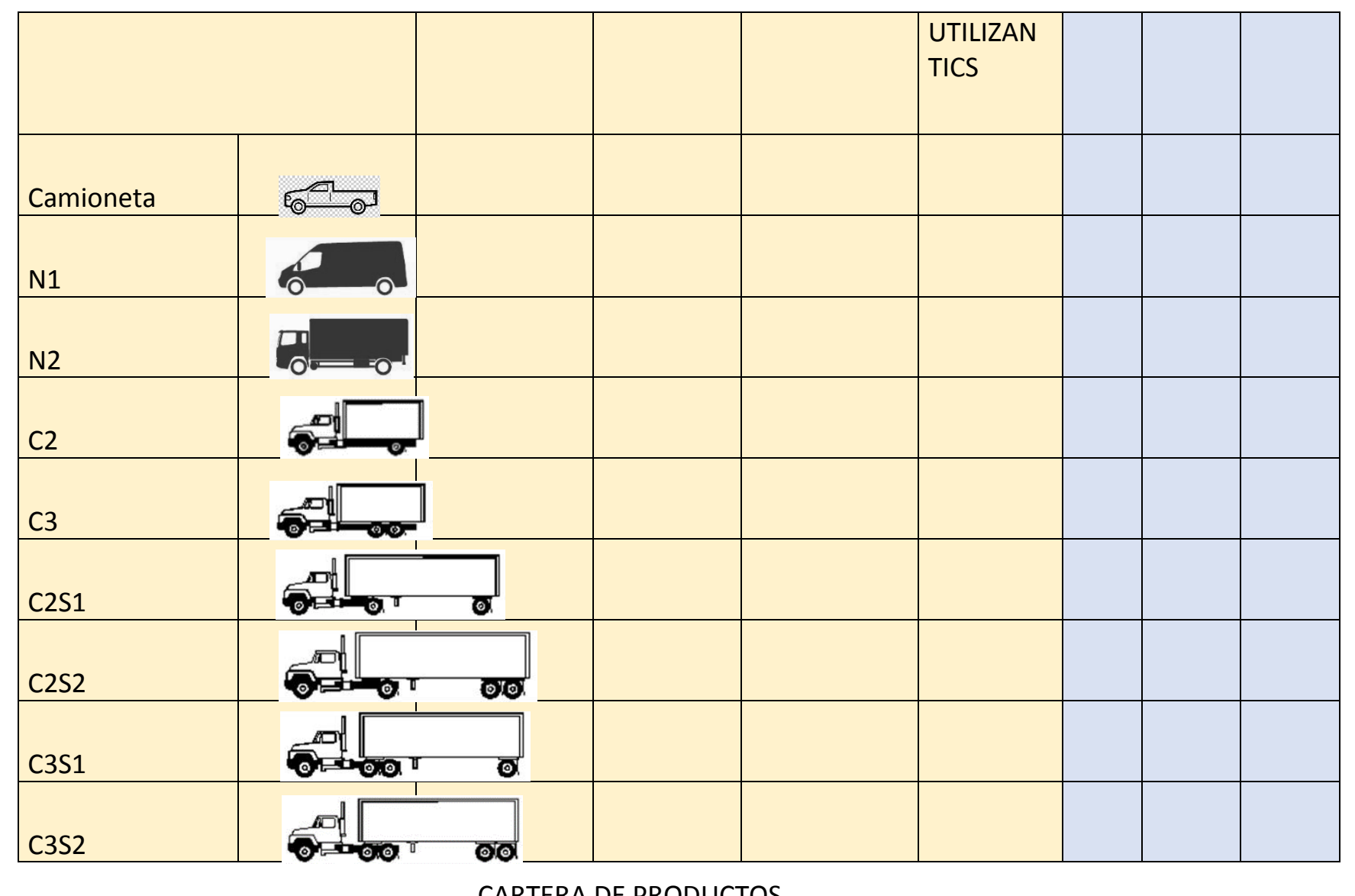

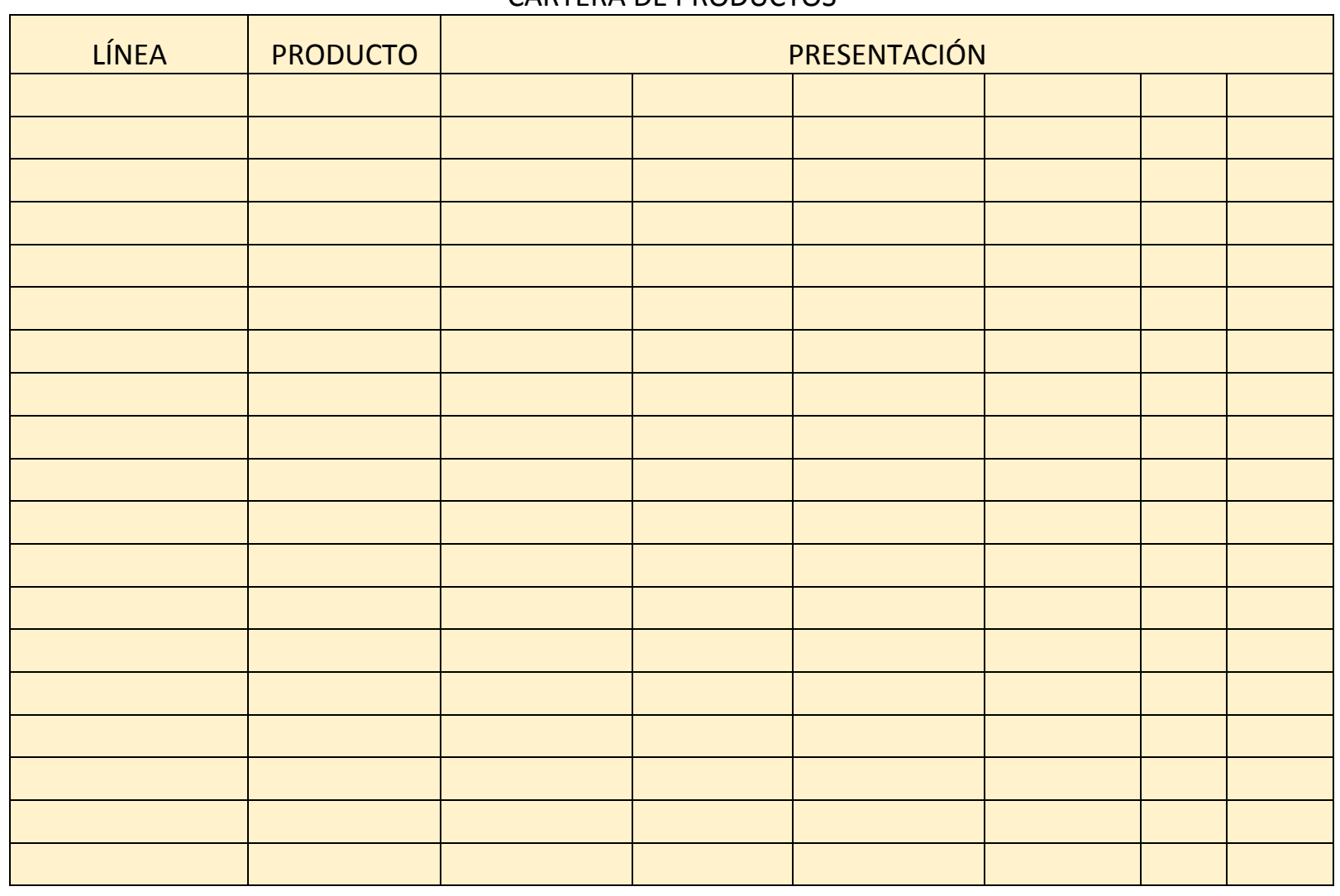

INFORMACIÓN DE LA GESTIÓN LOGÍSTICA 


\begin{tabular}{|l|l|}
\hline CÓDIGO/NOMB & \\
RE DE RUTA & \\
\hline NUMERO DE & \\
CLIENTES & \\
\hline FRECUENCIA & \\
\hline TIEMPO DE & \\
RECORRIDO & \\
ESTIMADO & \\
\hline TIEMPO DE & \\
CARGA & \\
\hline TIEMPO & \\
PROMEDIO DE & \\
DESCARGA & \\
\hline DIA DE & \\
RECORRIDO DE & \\
LA RUTA & \\
\hline HORA DE INICIO & \\
DE RECORRIDO & \\
\hline HORA DE & \\
FINALIZACIÓN & \\
DE RECORRIDO & \\
\hline KPI: ENTREGAS & \\
SATISFECHAS & \\
\hline KPI: ENTREGAS & \\
INSATISFECHAS & \\
\hline
\end{tabular}

\section{IDENTIFICACIÓN DE LA RUTA}

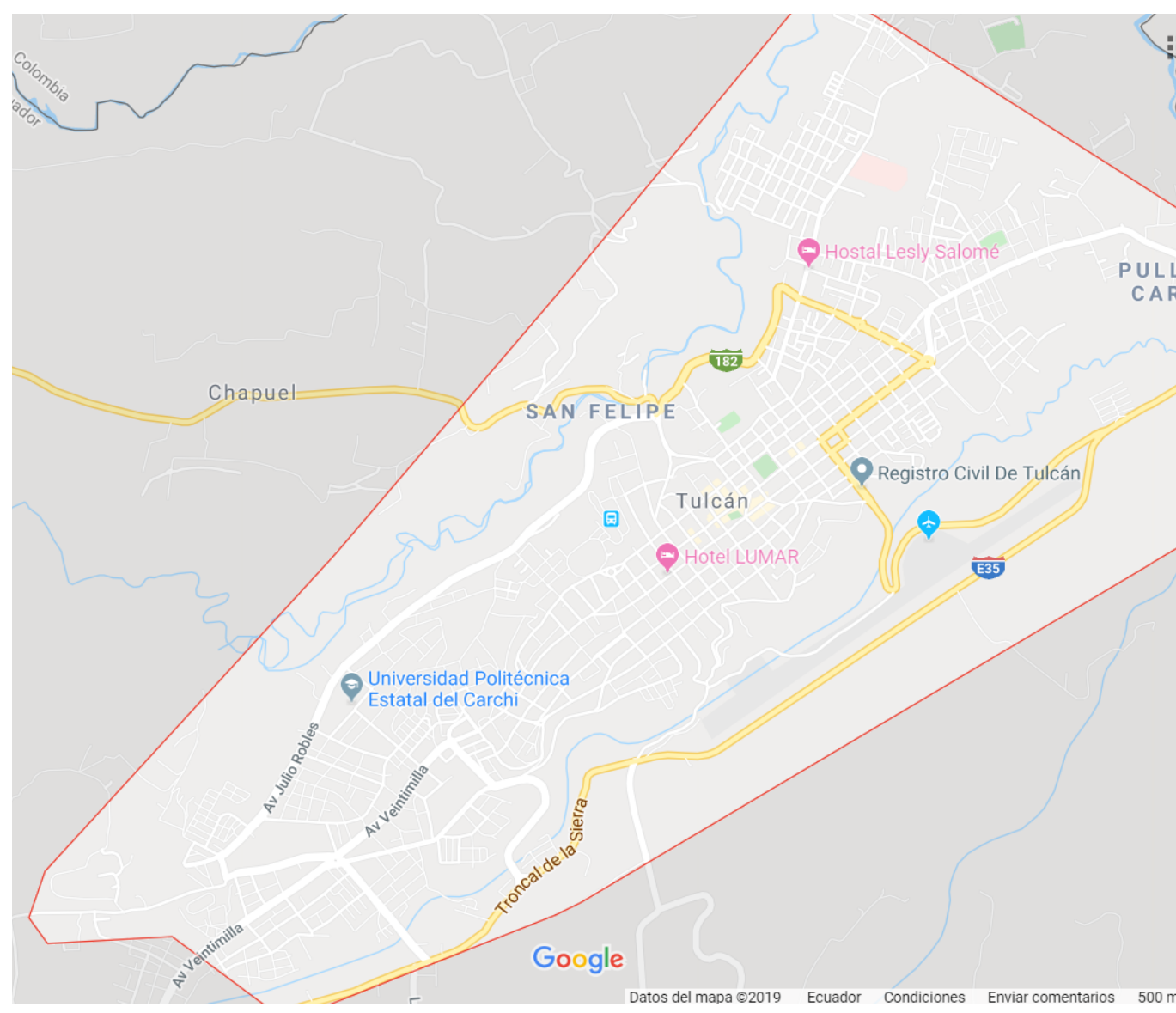

Cuadro 5

Matriz de recolección de datos (empresa ARCA)

\begin{tabular}{|c|l|r|r|r|c|}
\hline \multirow{2}{*}{ EMPRESA } & SECTOR & No VEHÍCULOS & \multicolumn{2}{|c|}{$\begin{array}{c}\text { DIAS DE } \\
\text { RECORRIDO }\end{array}$} & TIEMPO DE CARGA \\
\hline \multirow{3}{*}{ ARCACONTINENTAL } & NORTE & 2 & & LUNES Y SABADO & 1 H \\
\cline { 2 - 6 } & CENTRO & 2 & & LUNES Y SABADO & 1 H \\
\cline { 2 - 7 } & SUR & 1 & & LUNES Y SABADO & 1 H \\
\hline
\end{tabular}

TIENDAS VISITADAS

NORTE

\begin{tabular}{|l|l|c|c|c|c|}
\hline NOMBRE & DIRECCION & LATITUD & LONGITUD & TAMAÑO & $\begin{array}{c}\text { CANTIDAD DE } \\
\text { ABASTECIMIENTO }\end{array}$ \\
\hline MARIANITA & AV. 24 DE MAYO & 0,826514 & $-77,692261$ & GRANDE & 141,2 \\
\hline DON RAMIRO & SUCRE Y CHILE & 0,82041 & $-77,70682$ & PEQUEÑA & 27 \\
\hline GUADALUPE & SUCRE Y ARGENTINA & 0,82148 & $-77,70577$ & PEQUEÑA & 27 \\
\hline VIVERES NELLY & SUCRE Y ROBERTO GRIJALVA & 0,81951 & $-77,70803$ & PEQUEÑA & 27 \\
\hline LAURITA & SUCRE Y PANAMA & 0,81537 & $-77,71343$ & MEDIANA & 91,4 \\
\hline
\end{tabular}




\begin{tabular}{|c|c|c|c|c|c|}
\hline VIVERES CARMITA & $\begin{array}{l}\text { ADOLFO BECKER Y MANUEL } \\
\text { ACUÑA }\end{array}$ & 0,831544 & $-77,712835$ & GRANDE & 141,2 \\
\hline VIVERES SALITO & ADOLFO BECKER & 0,832935 & $-77,714876$ & MEDIANA & 91,4 \\
\hline VIVERES SABI & ADOLFO BECKER & 0,831841 & $-77,712833$ & GRANDE & 141,2 \\
\hline VIVERES MARY & AV. CORAL Y ARGENTINA & 0,821991 & $-77,706419$ & GRANDE & 141,2 \\
\hline VIVERES LUPITA & BRASIL Y ARGENTINA & 0,82367 & $-77,712692$ & PEQUEÑA & 27 \\
\hline VIVERES MARACAI & AV. FRANCISCO Y ARGENTINA & 8,524374 & 77,712605 & PEQUEÑA & 27 \\
\hline TIENDA DANIELITA & AV. SAN FRANCISCO & 0,82533 & 77,212235 & MEDIANA & 91,4 \\
\hline TIENDA LA HABANA & AV. SAN FRANCISCO Y ARGENTINA & 0,825143 & 77,712395 & GRANDE & 141,2 \\
\hline MINIMARKET GREEN & $\begin{array}{l}\text { PABLO ANIBAL VELA Y ERNESTO } \\
\text { NOVOA }\end{array}$ & 0,825832 & 77,710112 & GRANDE & 141,2 \\
\hline SAN CARLOS & $\begin{array}{l}\text { JUAN LEÓN MERA Y MEDARDO } \\
\text { ANGEL SILVA }\end{array}$ & 0,824841 & $-77,709692$ & PEQUEÑA & 27 \\
\hline SURTIDOS NANDOS & $\begin{array}{l}\text { JUAN RAMON JIMENEZ Y GABRIEL } \\
\text { MISTRAL }\end{array}$ & 0,830449 & $-77,712403$ & MEDIANA & 91,4 \\
\hline VIVERES DON BOLO & AV. RUBEN DARIO & 0,83094 & $-77,715272$ & MEDIANA & 91,4 \\
\hline VIVERES PANCHITO & VENEZUELA Y OLMEDO & 0.815319 & -7.771 .257 & MEDIANA & 91,4 \\
\hline VIVERES YOSHUA & OLMEDO Y VENEZUELA & 0.81536 & -77.712 .346 & GRANDE & 141,2 \\
\hline TIENDA NADITO & OLMEDO Y BOLIVIA & 0,816388 & $-77,710945$ & PEQUEÑA & 27 \\
\hline $\mathrm{S} / \mathrm{N}$ & MANABI Y BRASIL & 0.824192 & -77.710 .469 & PEQUEÑA & 27 \\
\hline $\begin{array}{l}\text { ABARROTES LA } \\
\text { ALBORADA }\end{array}$ & OLMEDO Y PAGUAY & 0.817552 & $-77,709395$ & MEDIANA & 91,4 \\
\hline VIVERES SAN FRANCISCO & OLMEDO Y ROBERTO GRIJALVA & 0,819877 & $-77,70781$ & MEDIANA & 91,4 \\
\hline VIVERES KALE & $\begin{array}{l}\text { AV. SAN FRANCISCO Y RUBEN } \\
\text { DARIO }\end{array}$ & 0,828957 & $-77,711478$ & PEQUEÑA & 27 \\
\hline TIENDA DOÑA TERE & & 0,835005 & $-77,70891$ & GRANDE & 141,2 \\
\hline MAIK & BARRIO 4 ESQUINAS & 0,836565 & $-77,705255$ & GRANDE & 141,2 \\
\hline VIVERES YOLITA & $\begin{array}{l}\text { AV. SAN FRANCISCO Y GUSTAVO } \\
\text { ADOLFO BECKER }\end{array}$ & 0,930593 & -77.710 .846 & PEQUEÑA & 27 \\
\hline $\mathrm{S} / \mathrm{N}$ & CIUDADELA SAN FRANCISCO & 0,828561 & -7.771 .303 & PEQUEÑA & 27 \\
\hline VIVERES SOILA & AV. CEMENTERIO Y MANABI & $0 ., 818109$ & $-77,713413$ & GRANDE & 141,2 \\
\hline VIVERES CRISTIAN & $\begin{array}{l}\text { AV. SAN FRANCISCO Y GABRIEL } \\
\text { AMISTRAL }\end{array}$ & 0,830864 & $-77,713272$ & PEQUEÑA & 27 \\
\hline VIVERES FLAQUITA & PABLO NERUDA & 0,830779 & $-77,713346$ & PEQUEÑA & 27 \\
\hline JUSTO AQUÍ & CALLE MANABI & 0,820986 & $-77,708596$ & PEQUEÑA & 27 \\
\hline VIVERES VALENTINA & $\begin{array}{l}\text { AV. SAN FRANCISCO Y CRESPO } \\
\text { TORAL }\end{array}$ & 0,827323 & $-77,710829$ & GRANDE & 141,2 \\
\hline VIVERES MAGALY & 24 DE MAYO & 0,82787 & $-77,699114$ & PEQUEÑA & 27 \\
\hline $\begin{array}{l}\text { MINIMARKET DIVINO } \\
\text { NIÑO }\end{array}$ & $\begin{array}{l}\text { AV. } 24 \text { DE MAYO Y NICOLAS } \\
\text { DELGADO }\end{array}$ & 0,825298 & $-77,690011$ & GRANDE & 141,2 \\
\hline VIVERES TIENDA CYBER & MANABÍ Y PARAGUAY & 0,809481 & $-77,711712$ & GRANDE & 141,2 \\
\hline VIVERES SAN FRANCISCO & 24 DE JULIO Y 6 DE DICIEMBRE & 0,818646 & $-77,706656$ & MEDIANA & 91,4 \\
\hline LOS CHAMOS & PARAGUAY Y MALDONADO & 0,816914 & $-77,709216$ & PEQUEÑA & 27 \\
\hline $\mathrm{S} / \mathrm{N}$ & VIA COMPLEJO PASTO & 0,836402 & $-77,704614$ & PEQUEÑA & 27 \\
\hline $\mathrm{S} / \mathrm{N}$ & PADRE PONCE Y ALFONCIN & 0,835173 & $-77,710588$ & MEDIANA & 91,4 \\
\hline VIVERES YARUNI & ARGENTINA Y MANABI & 0,823019 & $-77,707318$ & MEDIANA & 91,4 \\
\hline $\mathrm{S} / \mathrm{N}$ & CIUDADELA PADRE CARLOS & 0,832017 & $-77,715377$ & GRANDE & 141,2 \\
\hline
\end{tabular}




\begin{tabular}{|l|l|c|c|c|c|}
$\begin{array}{l}\text { ABASTOS MARIA } \\
\text { FERNANDA }\end{array}$ & $\begin{array}{l}\text { AV. CORAL ENTRE CHILE Y } \\
\text { ARGENTINA }\end{array}$ & 0,821317 & $-77,707058$ & GRANDE & 141,2 \\
\hline VIVERES CASTILLO & MANABI Y GUATEMALA & 0,82011 & $-77,706104$ & GRANDE & 141,2 \\
\hline VIVERES POZO & 10 DE AGOSTO Y LOJA & 0,801683 & $-77,720055$ & PEQUEÑA & 27 \\
\hline VIVERES SANTI & CIUDADELA SAN FRANCISCO & 0,828887 & $-77,713066$ & MEDIANA & 91,4 \\
\hline
\end{tabular}

TIENDAS VISITADAS

CENTRO

\begin{tabular}{|l|l|c|c|c|c|}
\hline MINIMARKET SALOME & PANAMA Y BOLIVIA & 0,815153 & $-77,710802$ & GRANDE & 141,2 \\
\hline DONDE CHICHO & MANABI Y URUGUAY & 0,82026 & $-77,71068$ & PEQUEÑA & 27 \\
\hline SU TIENDA & MANABI Y PARAGUAY & 0,819427 & $-77,711702$ & PEQUEÑA & 27 \\
\hline VIVERES ANGIE & ROCAFUERTE Y SUCRE & 0,811092 & $-77,7391$ & PEQUEÑA & 27 \\
\hline VIVERES MARGARITA & AV. VEINTIMILLA Y PATRONATO & 0,798855 & $-77,73344$ & MEDIANA & 91,4 \\
\hline VIVERES DANIS & BOYACA ENTRE BOLIVAR Y SUCRE & 0,814201 & $-77,716354$ & GRANDE & 141,2 \\
\hline LIDIA BENAVIDES & $\begin{array}{l}\text { RAFAEL ARELLANO Y CLEMENTE } \\
\text { BORJA }\end{array}$ & 0,808441 & $-77,725256$ & MEDIANA & 91,4 \\
\hline VIVERES ANDREITA & $\begin{array}{l}\text { RAFAEL ARELLANO Y GENERAL } \\
\text { LANDAZURI }\end{array}$ & 0,809895 & $-77,724291$ & PEQUEÑA & 27 \\
\hline VIVERES TAMYA & BOLIVAR Y PICHINCHA & 0,811966 & $-77,719306$ & PEQUEÑA & 27 \\
\hline MINMARKET BRITANY & CALLE CALDERON Y BOLIVIA & 0,817649 & $-77,71312$ & GRANDE & 141,2 \\
\hline COMERCIAL BAYMARY & ESMERALDAS Y 10 DE AGOSTO & 0,815863 & $-77,719889$ & GRANDE & 141,2 \\
\hline VIVERES SU VECINO & OLMEDO Y RICARDO DEL HIERRRO & 0,819268 & $-77,707293$ & MEDIANA & 91,4 \\
\hline $\begin{array}{l}\text { TIENDA FAMILIAR CDLA. } \\
\text { DEL MAESTRO }\end{array}$ & $\begin{array}{l}\text { LUIS FELIPE BORJA Y HERMANO } \\
\text { MIGUEL }\end{array}$ & 0,812399 & $-77,71129$ & PEQUEÑA & 27 \\
\hline
\end{tabular}

TIENDAS VISITADAS SUR

\begin{tabular}{|c|c|c|c|c|c|}
\hline$S / N$ & AV. VEINTIMILLA Y CAMILO PONCE & 0,795746 & $-77,737341$ & MEDIANA & 91,4 \\
\hline TIENDA DEL AHORRO & AV. VEINTIMILLA & 0,797392 & $-77,734752$ & GRANDE & 141,2 \\
\hline $\mathrm{S} / \mathrm{N}$ & LAS TEJERIAS Y GANADERO & 0,80256 & $-77,73192$ & PEQUEÑA & 27 \\
\hline$S / N$ & SUMACO Y ANTISANA & 0,805772 & $-77,73271$ & PEQUEÑA & 27 \\
\hline VIVERES SU TIENDA & GANADERO Y LAS TEJERIAS & 0,802504 & $-77,731694$ & PEQUEÑA & 27 \\
\hline $\mathrm{S} / \mathrm{N}$ & QUILINDAMA Y JULIO ROBLES & 0,80778 & $-77,73255$ & GRANDE & 141,2 \\
\hline VIVERES GIRASOL & AV. VEINTIMILLA & 0,794648 & $-77,738795$ & MEDIANA & 91,4 \\
\hline VIVERES PAOLITA & TEJERIAS Y PASAJE PULMA & 0,8016 & $-77,732412$ & PEQUEÑA & 27 \\
\hline MINIMARKET LEON & CORAZÓN Y GUAGUA PICHINCHA & 0,8016 & $-77,732412$ & MEDIANA & 91,4 \\
\hline VIVERES LILIBETH & CHACANA Y GUAGUA PICHINCHA & 0.801600 & $-77,732412$ & GRANDE & 141,2 \\
\hline VIVERES REGEL & $\begin{array}{l}\text { GUAGUA PICHINCHA Y SIERRA } \\
\text { NEGRA }\end{array}$ & 0,815678 & $-77,728$ & MEDIANA & 91,4 \\
\hline VIVERE GAELITO & AV. VEINTIMILLA Y POTOSI & 0,798121 & $-77,73932$ & MEDIANA & 91,4 \\
\hline $\mathrm{S} / \mathrm{N}$ & LAS TEJERIAS & 0,801778 & $-77,734097$ & GRANDE & 141,2 \\
\hline SUPER EXPRESS 60 & AV. VEINTIMILLA Y JUAN XXIIII & 0,801292 & $-77,73129$ & PEQUEÑA & 27 \\
\hline VIVERES JOSTIN & JOSE DE ANTEPARA & 0,799674 & $-77,741319$ & GRANDE & 141,2 \\
\hline VIVERES SU ECONOMIA & JESUS DEL GRAN PODER & 0,795702 & $-77,739283$ & PEQUEÑA & 27 \\
\hline
\end{tabular}




\begin{tabular}{|c|c|c|c|c|c|}
\hline VIVERES SOFIA & $\begin{array}{l}\text { FLORENCIA DE ALGARICOA Y JOSE } \\
\text { ANTEPARA }\end{array}$ & 0,798626 & $-77,74763$ & MEDIANA & 91,4 \\
\hline VIVERES ISRAEL & LORENZO Y JOSE DE ANTEPARA & 0,798626 & $-77,740761$ & PEQUEÑA & 27 \\
\hline VIVERES JORDAN & ECUADOR Y OLMEDO & 0,803845 & $-77,725103$ & PEQUEÑA & 27 \\
\hline DON PANCHO & ECUADOR Y GENERAL URBINA & 0,80525 & $-77,72648$ & GRANDE & 141,2 \\
\hline VIVERES CHICHARRITO & CALLE OLMEDO & 0,803403 & $-77,725497$ & GRANDE & 141,2 \\
\hline $\mathrm{S} / \mathrm{N}$ & AV. ANDRES VILLA Y TEJERIAS & 0,799317 & $-77,735105$ & GRANDE & 141,2 \\
\hline SAN BRASIL & $\begin{array}{l}\text { AV. VEINTIMILLA Y CALLE } \\
\text { PORTUGAL }\end{array}$ & 0,799796 & $-77,732374$ & MEDIANA & 91,4 \\
\hline VIVERES ECUADOR & BOLIVAR Y ECUADOR & 0,804356 & $-77,727215$ & GRANDE & 141,2 \\
\hline VIVERES ALBITA & $\begin{array}{l}\text { AV. VEINTIMILLA FRENTE AL } \\
\text { PATRONATO }\end{array}$ & 0,787348 & $-77,74398$ & MEDIANA & 91,4 \\
\hline VIVERES ROSITA & $\begin{array}{l}\text { AV. CENTENARIO Y CIUDADELA } \\
\text { DEL CHOFER }\end{array}$ & 0,802414 & $-77,728291$ & PEQUEÑA & 27 \\
\hline$S / N$ & $\begin{array}{l}\text { JOSE DE ANTEPARA Y JOSE DE } \\
\text { VARGAS }\end{array}$ & 0,799324 & $-77,739549$ & GRANDE & 141,2 \\
\hline EL CENTAVITO & ISIDRO PAVON Y JO SE BILTAMIN & 0,831138 & $-77,713827$ & PEQUEÑA & 27 \\
\hline MINIMARKET SURIMAX & $\begin{array}{l}\text { AV. TULCANAZA Y AV ANDRES } \\
\text { BELLO }\end{array}$ & 0,797258 & $-77,734198$ & PEQUEÑA & 27 \\
\hline CASTIPAN & BOLIVAR Y IMBABURA & 0,809 & $-77,72$ & GRANDE & 141,2 \\
\hline VIVERES YUPANQUI & SUCRE Y BOYACA & 0,813 & $-77,71$ & MEDIANA & 91,4 \\
\hline VIVERES LILIANA & SUCRE Y BOYACA & 0,813 & $-77,71$ & MEDIANA & 91,4 \\
\hline VIVERES PATTY & $\begin{array}{l}\text { AV. VEINTIMILLA Y GENERAL } \\
\text { CORDOBA }\end{array}$ & 0,793091 & $-77,740737$ & GRANDE & 141,2 \\
\hline$S / N$ & $\begin{array}{l}\text { AV. VEINTIMILLA Y GENERAL } \\
\text { CORDOBA }\end{array}$ & 0,79313 & $-77,741028$ & GRANDE & 141,2 \\
\hline MERCATODO & $\begin{array}{l}\text { AV. VEINTIMILLA Y GENERAL } \\
\text { SANTANDER }\end{array}$ & 0,793287 & $-77,740527$ & MEDIANA & 91,4 \\
\hline MINMARKET DEL SUR & AV. VEINTIMILLA Y ALION & 0,796933 & $-77,735411$ & GRANDE & 141,2 \\
\hline VIVERES PORTAL & AV. VEINTIMILLA Y EL PORTAL & 0,394415 & -77.739 .029 & PEQUEÑA & 27 \\
\hline VIVERES SANDRITA & SAN CRISTOBAL TEJERIAS & 0,814307 & $-77,721582$ & GRANDE & 141,2 \\
\hline VIVERES TERESITA & $\begin{array}{l}\text { VEINTIMILLA Y PASAJE } \\
\text { ATAHUALPA }\end{array}$ & 0,802825 & $-77,730036$ & MEDIANA & 91,4 \\
\hline $\begin{array}{l}\text { LAS DELICIAS DEL } \\
\text { PALADAR }\end{array}$ & $\begin{array}{l}\text { AV. VEINTIMILLA A 20M DEL } \\
\text { TERMINAL }\end{array}$ & 0,803729 & $-77,728247$ & MEDIANA & 91,4 \\
\hline LA PASTUSITA & AV.UNIVERSITARIA Y ANTISANA & 0,804928 & $-77,7381$ & PEQUEÑA & 27 \\
\hline VIVERES SAHIR & BOLIVAR Y CARABOBO & 0,805016 & $-77,726581$ & PEQUEÑA & 27 \\
\hline S.N & AV. EL SEMINARIO Y LORENZO & 0,796931 & $-77,737688$ & MEDIANA & 91,4 \\
\hline LA REBAJA & JULIO ROBLES Y PASAJE NOBOA & 0,798586 & $-77,739394$ & MEDIANA & 91,4 \\
\hline VIVERES ARITO & RAFAEL ARELLANO Y CARABOBO & 0,805074 & $-77,727125$ & PEQUEÑA & 27 \\
\hline VARIEDADES ICDL & AV. VEINTIMILLA Y MERINO & 0,794357 & $-77,739012$ & MEDIANA & 91,4 \\
\hline VIVERES BRITANY & $\begin{array}{l}\text { AV.VEINTIMILLA JUNTO AL } \\
\text { TERMINAL HUACA JULIO A }\end{array}$ & 0,803531 & -728812 & MEDIANA & 91,4 \\
\hline VIVERES LA UNIVERSAL & $\begin{array}{l}\text { AV. VEINTIMILLA JUNTO AL } \\
\text { TERMINAL HUACA JULIO A }\end{array}$ & 0,803531 & $-77,728735$ & GRANDE & 141,2 \\
\hline S.N & MIRA Y ESPEJO & 0,799476 & $-77,727478$ & MEDIANA & 91,4 \\
\hline S.N & $\begin{array}{l}\text { AV- VEINTIMILLA Y SAN } \\
\text { CRISTOBAL }\end{array}$ & 0,798487 & $-77,733877$ & MEDIANA & 91,4 \\
\hline CABINAS DOMECELL & AV.VEINTIMILLA Y JUAN XXIII & 0,801284 & $-77,731068$ & PEQUEÑA & 27 \\
\hline
\end{tabular}




\begin{tabular}{|c|c|c|c|c|c|}
\hline VIVERES NASLY & LAS TEJERIAS Y LAGUNA NEGRA & 0,801619 & $-77,73235$ & GRANDE & 141,2 \\
\hline LA ECONÓMICA & AV.VEINTIMILLA Y SAN CRISTOBAL & 0,798366 & $-77,733887$ & PEQUEÑA & 27 \\
\hline GABRIELITA & ISIDRO PAVON Y JOSE DE VILLAMIL & - & - & MEDIANA & 91,4 \\
\hline S.N & $\begin{array}{l}\text { AV. JULIO ROBLES Y JOSE DE } \\
\text { ANTEPARA }\end{array}$ & - & - & PEQUEÑA & 27 \\
\hline PETRONA & $\begin{array}{l}\text { JOSE DE ANTENARA Y DIEGO DE } \\
\text { NOVOA }\end{array}$ & - & - & GRANDE & 141,2 \\
\hline POLLO CRIOLLO & AV.VEINTIMILLA & - & - & MEDIANA & 91,4 \\
\hline LAS GRADAS & BOLIVAR Y LAS GRADAS & - & - & GRANDE & 141,2 \\
\hline COMERCIAL CRIS & BOLIVAR Y GENERAL PLAZA & - & - & PEQUEÑA & 27 \\
\hline AILIN & ITALIO Y SUECIA & - & - & MEDIANA & 91,4 \\
\hline MINI MARKET & AV. VEINTIMILLA Y JUAN 23 & - & - & GRANDE & 141,2 \\
\hline MISELANEA NARLAG & TEJERIAS Y ANDRES BELLO & - & - & PEQUEÑA & 27 \\
\hline LA TIENDA & AV. VEINTIMILLA Y PORTUGAL & - & - & PEQUEÑA & 27 \\
\hline L.B.R.G & SANGAI Y ITALO & - & - & PEQUEÑA & 27 \\
\hline DESPENSA & AV. VEINTIMILLA & - & - & PEQUEÑA & 27 \\
\hline S.N & AV. VEINTIMILLA & - & - & MEDIANA & 91,4 \\
\hline VIVERES DON SANTI & $\begin{array}{l}\text { VICENTE DE LA CORREA Y } \\
\text { GONZALO ARAUJO }\end{array}$ & - & - & PEQUEÑA & 27 \\
\hline VIVERES MADELAINEE & AV. TULCANAZA Y MIRA & 0,798631 & $-77,729673$ & MEDIANA & 91,4 \\
\hline VIVERES MARTITA & MIRA & 0,798206 & $-77,72973$ & GRANDE & 141,2 \\
\hline VIVERES ANDRESITO & $\begin{array}{l}\text { CELIN ARELLANO, ESPEJO Y PIO } \\
\text { MONTUFAR }\end{array}$ & & & MEDIANA & 91,4 \\
\hline VIVERES POZO & JOSE FELIX Y CELIN ARELLANO & 0,797322 & $-77,728238$ & MEDIANA & 91,4 \\
\hline VIVERES ELIZABETH & AV TULCANZA Y MIRA & 0,798136 & $-77,729592$ & PEQUEÑA & 27 \\
\hline J Y M MINIMARKET & BOLIVAR Y CARABOBO & 0,805 & $-77,72$ & PEQUEÑA & 27 \\
\hline VIVERES BENAVIDES & BOLIVAR Y GENERAL LANDAZURI & 0,808183 & $-77,723532$ & GRANDE & 141,2 \\
\hline VIVERES ESTEFANNY & LAS TEJERIAS Y MOJANDA & 0,80177 & $-77,732445$ & GRANDE & 141,2 \\
\hline S.N & LAS TEJERIAS Y MEXICO & 0,801439 & $-77,73238$ & MEDIANA & 91,4 \\
\hline SRA LILIANA CHALA & $\begin{array}{l}\text { JOSE DE ANTEPAN Y DIEGO } \\
\text { NOVOA }\end{array}$ & 0,798515 & $-77,730132$ & PEQUEÑA & 27 \\
\hline VIVERES SAMI & $\begin{array}{l}\text { AV. JULIO ROBLES Y JOSE DE } \\
\text { ANTEPANA }\end{array}$ & 0,79899 & $-77,738887$ & PEQUEÑA & 27 \\
\hline MEYESTI & $\begin{array}{l}\text { AV VENTIMILLA Y JUAN RAMON } \\
\text { ARELLANO }\end{array}$ & 0,794365 & $-77,73906$ & GRANDE & 141,2 \\
\hline VIVIERES CAMPO & AV VENTIMILLA & 0,795129 & $-77,738276$ & GRANDE & 141,2 \\
\hline VIVERES ANITA & AV VENTIMILLA & 0,796397 & $-77,73618$ & PEQUEÑA & 27 \\
\hline VIVIERES RUMIÑAHUI & AV VEINTIMILLA Y CAMILO PONCE & 0,795668 & $-77,737498$ & MEDIANA & 91,4 \\
\hline VIVERES & AV ANDRES BELLO E INGLATERRA & 0,794832 & $-77,732558$ & MEDIANA & 91,4 \\
\hline $\mathrm{S} / \mathrm{N}$ & CARLOS OÑA Y ALFONSO HERRERA & 0,79598 & $-77,735266$ & GRANDE & 141,2 \\
\hline SU TIENDA & VIAS LAS PALMAS & - & - & PEQUEÑA & 27 \\
\hline
\end{tabular}

Fuente: Elaboración propia

Esta obra está bajo una Licencia Creative Commons Attribución-NoCommercial 4.0 International

(cc) BY-NC 